\title{
EFFECT OF MORINGA LEAVES EXTRACTS AGAINST POISONED BY PB IN RATS
}

\author{
K.E. Hussain, Samia M. Khaleil and F. A. Abd EL-Salam \\ Department of Biochemistry, Faculty of Agriculture, Menoufia University, Egypt.
}

Received: Dec. 2, 2018

Accepted: Dec. 8, 2018

\begin{abstract}
This study was carried out to evaluate the effect of Moringa leaves extracts for treatment the toxicity by Lead in rats. Lead toxicity often to various clinical conditions, Batteries, power cables, computer, water pipes, found in petroleum oil, coal and tobacco. Moringa leaves were found to contain antioxidants. $(237.1 \mathrm{mg} / \mathrm{g})$ phenols and $(141.7 \mathrm{mg} / \mathrm{g})$ flavones. Phenols were found to contain 23 phenolic compounds, vanillic, salicylic, and Catechins phenols are present in large amounts. Exposure to Lead increased the activity of liver enzymes AST, ALT and ALP while the level of albumin was decreased there is a significant increase in the level of urea and creatinine. The addition of the water and acetone extracts reduced the harmful effect of lead poisoning the treatment with Moringa leaf extracts improved the activity of antioxidant parameters (SOD,CAT and MDA), and decrease in the accumulation of lead in both liver and brain in the experimental rats.
\end{abstract}

Key words: Moringa leaves Poisoned by pb.

\section{INTRODUCTION}

Environmental pollution is the presence of a pollutant in the environment such as air, water, soil and consequently in food which may be poisonous or toxic and will cause harm to living things in the polluted environment. Lead affects every one of the body's organ systems, especially the nervous system but also the bones, teeth, the kidneys, the cardiovascular, immune and reproductive systems (White et al 2007). Lead has been used widely since 5000 BC for application in metal products, pipelines, cables, as well as in paints and pesticides Adhikari, et al., (2001)

This abundant ecological pollutant enters the atmosphere via fabrication of coal, oil, iron, batteries, and steel, and also from smelters, refuge, and tobacco smoke. It can enter the human body via uptake of food (65\%), water (20\%) and air (15\%) Clayton et al., (2002).
Target organs affected by lead are bones, brain, blood, kidneys and thyroid glands Shalan et al., (2005).

Moringa oleifera, is the new challenge of tradition medical plant use both in vitro and in vivo for reducing metal toxic in environment and living organism. (Paliwal et al., 2011).

Moringa provides a rich and rare combination of nutrients, amino acids, antioxidants, antiaging and antiinflammatory properties used for nutrition and healing. The particular plant family is rich in a fairly unique group of glycoside compounds called glucosinolates and isothiocyanates. Small proteins/ peptides were isolated from the leaves of Moringa oleifera possessing antifungal and antibacterial activity (Dahot, 1988).

\section{MATERIALS AND METHODS.}

1- Leaves of Egyptian Moringa Oleifera were collected from Elmedoran, Elmesharak Keblly, Senoris, Faiom, 
Egypt in February.The Leaves were dry and grounded into powder.

2- Chemical composition.

2.1. Crude protein was determined (dry basis) according to the modified micro-Kjeldahl (Pirjo, and Pekka, 1996).

2.2 Crude Lipid, Moisture content, ash and crud fiber were determined according to A.O.A.C. (2000).

2.3 Total carbohydrate determined by difference $=100-($ ash $\%+$ Protein $\%+$ Fat\%).

2.4 Total phenolic compounds was Extraction and determination according to the method described by Daniel, and George, (1972). Free phenolic compounds in the ethanolic extract was determined colorimetrically by the method of Folin as described by (Gulcin, et al., 2002).

2.5 Extraction and determination of flavonoid compounds using the method reported by Dewanto et al., (2002).

2.6. Quantitative identification of phenolics and flavonoids by HPLC.

According to the modified method reported by Zuo, et al., (2002).

2.7 Reducing power (Ferric chloride method)

A spectrophotometric method (Oyaizu 1986) was used for the measurement of reducing power.

\section{Experimental animals.}

Adult male albino rats $(180 \pm 20 \mathrm{~g})$ were kept in plastic cages under hygienic for two weeks to acclimatized to laboratory conditions. The rats were fed on diets consisting of carbohydrates as starch $80 \%$, protein as Casein $10 \%$, cotton seed oil $5 \%$ salts mixture $4 \%$, Hegested et al., (1941). And vitamins mixture 1\% Schneeman et al., (1989). The animals were divided into one regime of them consists of 6 groups, each having 5 rats as follows (for 30 days). The first group was kept without any treatments as (Negative control), fed on standard diet. The second group were kept without any treatments as control fed on standard diet plus (CH3.coo) 2pb 4.4 $\mathrm{mg} / \mathrm{kg}$.bw. Positive $\mathrm{Pb}$ control. The third and for groups :rats were allowed to feed on standard diet plus Aqueous extract of Moringa Oleifera leaves at dose of $\mathbf{3 0 0}$ and $600 \mathrm{mg} / \mathrm{kg} \mathrm{b.w.} \mathrm{orally} \mathrm{respectively.}$

Groups 5 and 6 were feed on standard diet plus Acetone extract of. M. Oleifera leaves at doses 300 and $600 \mathrm{mg} / \mathrm{kg} \mathrm{bw}$. Orally respectively.

The experimental animals were killed by decapitation at the end of 30 days, then blood samples were collected and subjected to plasma separation.

\section{Liver function:}

A) Aspartate transaminase (AST) and Alanine transaminase (ALT) activity were determined in plasma by enzymatic colorimetric method as described by Young (1990).

B) Alkaline phosphatase (ALP) activity was determined colorimetrically as described by Moss, et al (1987).

C) Albumin (Alb) Concentration was determined as described by Canon et al (1974).

\section{Kidneys function:}

Urea and creatinine were determined as described by Young (2001).

\section{Oxidative stress parameters:}

- Superoxide dismutases (SODs) activity was determined in plasma as described by (Nishikimi et al., 1972).

- Catalase activity was determined in plasma as described by (Aebi 1984 ).

- Lipid peroxidation (LPO) level: determining the concentration of malondialdehyde (MDA) as described by Ohkawa et al., (1979).

Statistical analysis was done using the analysis variance according to Landue and Everitt (2004). 


\section{RESULTS AND DISCUSSION}

The moringa oliefera leaves contain total ash $7.98 \%$, crude lipid $3.22 \%$, crude protein $25.86 \%$, crude fiber $16.11 \%$, moisture $6.01 \%$ and total carbohydrates $40.83 \%$.The results are in accordance with those of Borges, et al., (2014).

They refer that moringa leaves chemical composition is complex, total ash $7.1 \%$, total lipids $4.7 \%$, crude protein $25.0 \%$, crude fiber $19.9 \%$, moisture $9.0 \%$ and total carbohydrates $43.9 \%$.

Total phenolics and total flavonoids contents in watery extracts for the leaves were $237.1 \mathrm{mg} / \mathrm{g}$ and $141.7 \mathrm{mg} / \mathrm{gbw}$ respectively.
HPLC analysis of the ethanolic extract for the phenolic and flavonoids compounds (Table 1) showed the presence of 23 phenolic compounds which were varied in their amounts. Ten of thime are found in high levels, they are e-vanillic, Pyrogallol, Salycilic, Catechin, P-OH-benzoic, Ellagic, Alpha-coumaric, 3,4,5-methoxy-cinnamic, Protocatechuic and Chlorogenic, its amounts ranged between 1348.52 to $154.38 \mathrm{mg} / 100 \mathrm{gm}$ b.w. mean, Catechol, Caffeine, Caffeic, Epicatechin and Ferulic were found in moderate amounts. Rinsed between 131.51 - $108.41 \mathrm{mg} / 100 \mathrm{~g}$ dry wit. Coumarin, Benzoic, Iso-ferulic, Vanillic, Gallic, Cinnamic, 4-aminobenzoic and Pcoumaric were found in low amounts.

Table (1): HPLC analysis of polyphenolics and flavonoids in of moringa leaves.

\begin{tabular}{|l||c||l||c||}
\hline \multicolumn{1}{|c|}{ Phenolic compounds } & $\begin{array}{c}\text { Mg I 100g } \\
\text { D.W }\end{array}$ & \multicolumn{1}{c|}{ flavonoids } & $\begin{array}{c}\text { Mg I 100g } \\
\text { D.W }\end{array}$ \\
\hline \hline Pyrogallol & 739.22 & Luteo.6-arabinose 8-glucose & 214.22 \\
\hline \hline Gallic & 41.81 & Luteo.6glocose 8-arabinose & 3.71 \\
\hline \hline - Amino-benzoic & 11.21 & Apig. 6-rhamnose 8-glucose & 61.13 \\
\hline \hline Protocatechuic & 159.43 & Apig. 6-glucose 8- rhamnose & 34.25 \\
\hline \hline Catechin & 424.93 & Naringin & 24.14 \\
\hline Catechol & 131.51 & Luteolin & 49.71 \\
\hline \hline Chlorogenic & 154.38 & Hesperidin & 167.38 \\
\hline Epicatechin & 110.35 & Rutin & 3.94 \\
\hline P-OH-benzoic & 219.84 & Quercetrin-3-O-glucoside & 26.39 \\
\hline \hline Caffeine & 121.59 & Rosmarinic & 131.04 \\
\hline \hline Caffeic & 118.94 & Apig.7-O-neohespiroside & 5.14 \\
\hline \hline Vanillic & 54.92 & Kamp.3,7-dirhamoside & 12.14 \\
\hline \hline P-coumaric & 10.89 & Apig.7-glucose & 20.83 \\
\hline \hline Ferulic & 108.41 & Quercetrin & 29.84 \\
\hline \hline Iso-ferulic & 68.37 & Quercetin & 2.41 \\
\hline \hline e-vanillic & 1348.52 & $\begin{array}{l}\text { Kaemp.3,(2-p-comaroyl) } \\
\text { glucose }\end{array}$ & 53.18 \\
\hline \hline Ellagic & 215.21 & Naringenin & 5.01 \\
\hline Alpha-coumaric & 192.17 & Hesperetin & 28.71 \\
\hline \hline Benzoic & 79.23 & Kampferol & 6.84 \\
\hline Coumarin & 84.93 & Rhamnetin & 5.03 \\
\hline 3,4,5-methoxy-cinnamic & 184.51 & Apigenin & 4.35 \\
\hline Salycilic & 593.82 & Acacetin & 19.01 \\
\hline \hline cinnamic & 19.71 & & \\
\hline \hline
\end{tabular}


Data in Table (1) showed that there are 22 components of flavonoids. Three of theme are found in high levels Luteo-6arabinose 8-gloucose 214.22, Hesperidin 167.38 and Rosmarinic 131.04.

In vitro antioxidant activity (reducing power assay).

Data in Fig (1) showed that the reducing power of moringa leaves watery extracts $(2.5 \%$ and $5 \%) /$ were 36.1 and $199.2 \mathrm{mmol}$ Ascorbic Eq, while such parameter for $(2.5 \%$ and $5 \%)$ acetone extracts were 23.41 and $152.31 \mathrm{mmol}$ Ascorbic Eq. The result are in accordance with that of Hossain, et al., (2012) who found that reducing power was (53.925 $\pm 5.25 \mathrm{mmol}$ Ascorbic Eq) for moringa leaves ethanol extract and it was(50.675 $\pm 3.699 \mathrm{mmol}$ Ascorbic Eq) for methanol extract of the same sample.

- Effect of moringa leaves extracts against Toxicity of $\mathrm{Pb}$ in rats.

- Effect of moringa leaves extracts on liver function test in rats against $\mathrm{Pb}$ Toxicity.

- From the data presented in Table (2)

- Data indicated that AST Activity were 20.0 and $19.0 \mathrm{U} / \mathrm{L}$ at zero time for negative and positive controls respectively. After 30 days, AST activity reached to 35 and $150 \mathrm{U} / \mathrm{L}$ for the two former controls.

- The addition of one of 300 and 600 $\mathrm{mg} / \mathrm{kg}$ watery extracts to rats poisoned by $\mathrm{Pb}$ revealed significant reduction in AST and ALT comparing with Positive controls.

- The data in Table (2) show that $(600$ $\mathrm{mg} / \mathrm{kg}$ ) aqueous extracts group exhibited the highest effect (55 U/L AST) and (50 U/L ALT) comparing with all treated groups.

- AST I ALT ratio Table (2) showed marked increases for all treatments (20 and 30 days) comparing with positive control. The AST I ALT ratio recorded values under 5 (between 1.02 and 1.07) this proved that the harmful of induced diabetes affect liver but not heart (ratio more than 20) as reported by Murray et al., (2006).

Data in Table (2) show that The addition of one of 300 and $600 \mathrm{mg} / \mathrm{kg}$ watery or 300 and $600 \mathrm{mg} / \mathrm{kg}$ acetone extracts to rats poisoned by $\mathrm{Pb}$ revealed significant reduce in ALP activity which recorded 225, 192, 231 and 210 U/L after 30 days of treatment respectively comparing with Positive control. (360 U/L).

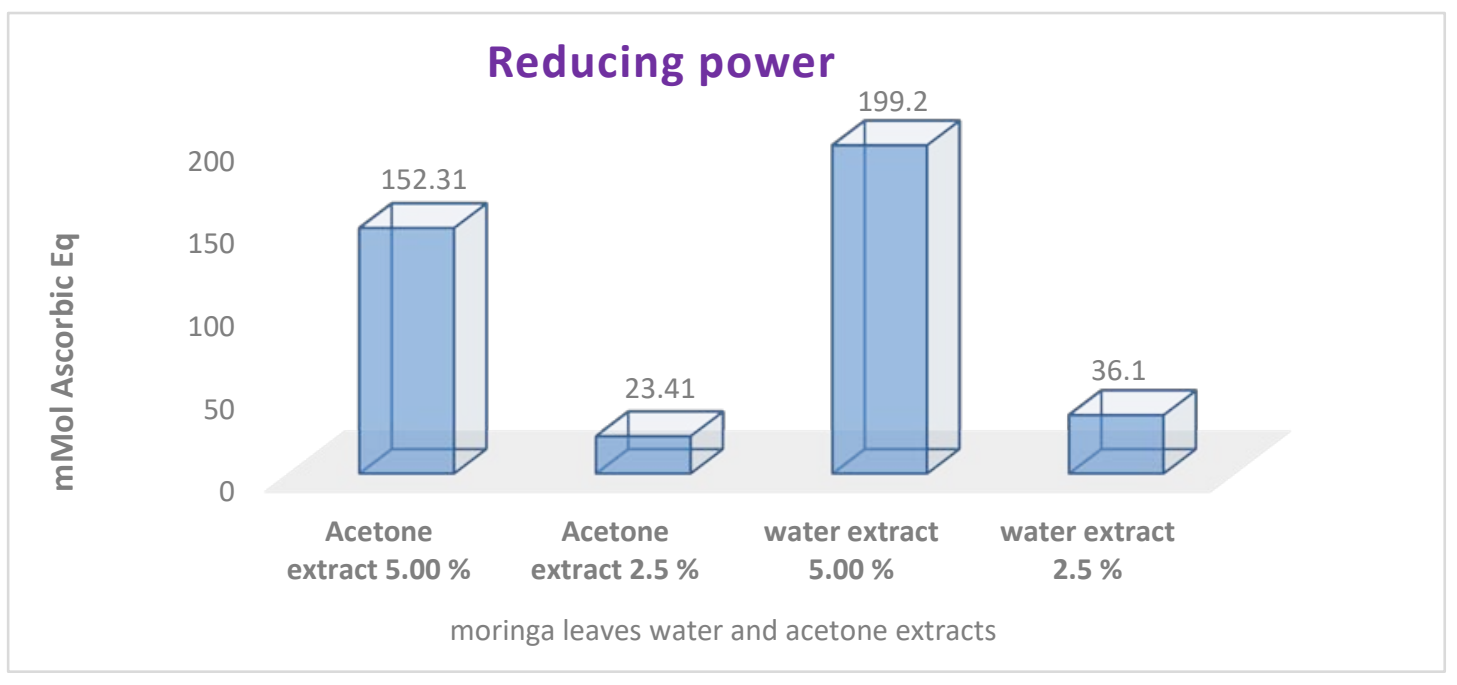

Fig (1): Reducing power activity for moringa leaves extracts 
Table (2) Effect of moringa leaves extracts against liver function in rats poisoned by $\mathrm{Pb}$

\begin{tabular}{|c|c|c|c|c|c|c|c|c|c|c|c|c|c|}
\hline & \multirow{2}{*}{ Exp. Period } & \multicolumn{2}{|c|}{$\begin{array}{c}\text { (I) } \\
\text { Negative control }\end{array}$} & \multicolumn{2}{|c|}{$\begin{array}{c}\text { ( II ) } \\
\text { Positive control }\end{array}$} & \multicolumn{2}{|c|}{$\begin{array}{c}\text { ( III ) } \\
\text { water extract } \\
300 \mathrm{mg} / \mathrm{kg} \mathrm{bw}\end{array}$} & \multicolumn{2}{|c|}{$\begin{array}{c}\text { ( IV ) } \\
\text { water extract } \\
600 \mathrm{mg} / \mathrm{kg} \mathrm{bw}\end{array}$} & \multicolumn{2}{|c|}{$\begin{array}{c}(\mathrm{V}) \\
\text { acetone extract } \\
300 \mathrm{mg} / \mathrm{kg} \mathrm{bw}\end{array}$} & \multicolumn{2}{|c|}{$\begin{array}{c}\text { ( VI ) } \\
\text { acetone extract } \\
600 \mathrm{mg} / \mathrm{kg} \mathrm{bw}\end{array}$} \\
\hline & & Result & $\%$ & Result & $\%$ & Result & $\%$ & Result & $\%$ & Result & $\%$ & Result & $\%$ \\
\hline \multirow{4}{*}{ 占 } & Zero Time & $20^{a} \pm 1.14$ & $100 \%$ & $19^{a} \pm 2.4$ & $95 \%$ & $21^{b} \pm 1.00$ & $105 \%$ & $23^{c} \pm 1.00$ & $115 \%$ & $20^{\mathrm{a}} \pm 1.94$ & $100 \%$ & $21^{b} \pm 2.00$ & $100 \%$ \\
\hline & 10 days & $27^{a} \pm 0.83$ & $100 \%$ & $47^{c} \pm 0.83$ & $174 \%$ & $42^{d} \pm 1.00$ & $155 \%$ & $35^{b} \pm 1.00$ & $129 \%$ & $43^{d} \pm 0.83$ & $159 \%$ & $40^{c} \pm 0.83$ & $148 \%$ \\
\hline & 20 days & $32^{a} \pm 1.22$ & $100 \%$ & $97^{f} \pm 1.92$ & $303 \%$ & $64^{\mathrm{d}} \pm 1.51$ & $200 \%$ & $43^{b} \pm 1.14$ & $134 \%$ & $70^{c} \pm 0.83$ & $218 \%$ & $56^{c} \pm 1.14$ & $175 \%$ \\
\hline & 30 days & $35^{\mathrm{a}} \pm 0.83$ & $100 \%$ & $150^{f} \pm 5.1$ & $428 \%$ & $75^{d} \pm 1.14$ & $214 \%$ & $55^{b} \pm 1.14$ & $157 \%$ & $80^{c} \pm 1.14$ & $228 \%$ & $61^{c} \pm 1.14$ & $174 \%$ \\
\hline \multirow{4}{*}{$\stackrel{5}{\rightleftarrows}$} & Zero Time & $18^{\mathrm{a}} \pm 1.14$ & $100 \%$ & $19^{b} \pm 1.48$ & $105 \%$ & $19^{b} \pm 1.81$ & $105 \%$ & $20^{c} \pm 2.3$ & $111 \%$ & $20^{c} \pm 0.89$ & $111 \%$ & $20^{c} \pm 1.51$ & $111 \%$ \\
\hline & 10 days & $25^{\mathrm{a}} \pm 1.51$ & $100 \%$ & $44^{c} \pm 0.70$ & $176 \%$ & $40^{d} \pm 1.00$ & $160 \%$ & $32^{b} \pm 1.48$ & $128 \%$ & $41^{d} \pm 1.14$ & $164 \%$ & $37^{c} \pm 1.48$ & $148 \%$ \\
\hline & 20 days & $28^{a} \pm 1.51$ & $100 \%$ & $94^{f} \pm 1.00$ & $335 \%$ & $62^{d} \pm 2.54$ & $248 \%$ & $41^{b} \pm 1.92$ & $149 \%$ & $68^{c} \pm 2.16$ & $241 \%$ & $55^{c} \pm 1.14$ & $196 \%$ \\
\hline & 30 days & $32^{a} \pm 1.14$ & $100 \%$ & $144^{f} \pm 1.8$ & $450 \%$ & $70^{d} \pm 1.3$ & $218 \%$ & $50^{b} \pm 0.7$ & $157 \%$ & $73 c \pm 2.7$ & $228 \%$ & $56^{c} \pm 1.81$ & $175 \%$ \\
\hline \multirow{4}{*}{$\frac{5}{\stackrel{5}{5}}$} & Zero Time & $1.05 \pm 0.03$ & $100 \%$ & $1.05 \pm 0.04$ & $95 \%$ & $1.04 \pm 0.02$ & 99\% & $1.04 \pm 0.04$ & $99 \%$ & $1.00 \pm 0.04$ & $95 \%$ & $1.05 \pm 0.02$ & $100 \%$ \\
\hline & 10 days & $1.00 \pm 0.04$ & $100 \%$ & $1.02 \pm 0.02$ & $102 \%$ & $1.02 \pm 0.01$ & $102 \%$ & $1.05 \pm 0.017$ & $105 \%$ & $1.02 \pm 0.02$ & $102 \%$ & $1.02 \pm 0.02$ & $102 \%$ \\
\hline & 20 days & $1.06 \pm 0.03$ & $100 \%$ & $1.03 \pm 0.01$ & $97 \%$ & $1.01 \pm 0.01$ & $96 \%$ & $1.02 \pm 0.02$ & $96 \%$ & $1.02 \pm 0.01$ & $96 \%$ & $1.01 \pm 0.01$ & $95 \%$ \\
\hline & 30 days & $1.05 \pm 0.02$ & $100 \%$ & $1.02 \pm 0.01$ & $97 \%$ & $1.05 \pm 0.01$ & $100 \%$ & $1.05 \pm 0.02$ & $100 \%$ & $1.06 \pm 0.01$ & $101 \%$ & $1.06 \pm 0.02$ & $101 \%$ \\
\hline \multirow{4}{*}{$\frac{2}{\frac{2}{\alpha}}$} & Zero Time & $92^{\mathrm{a}} \pm 1.3$ & $100 \%$ & $92^{\mathrm{a}} \pm 2.68$ & $100 \%$ & $91^{b} \pm 1.51$ & $99 \%$ & $93^{b} \pm 1.78$ & $101 \%$ & $90^{b} \pm 2.16$ & $97 \%$ & $92^{c} \pm 2.4$ & $100 \%$ \\
\hline & 10 days & $93^{a} \pm 1.34$ & $100 \%$ & $159^{f} \pm 3.3$ & $170 \%$ & $125^{\mathrm{d}} \pm 1.30$ & $134 \%$ & $115^{b} \pm 1.67$ & $123 \%$ & $132^{\mathrm{c}} \pm 1.58$ & $141 \%$ & $121^{c} \pm 1.67$ & $130 \%$ \\
\hline & 20 days & $95^{\mathrm{a}} \pm 1.14$ & $100 \%$ & $240^{f} \pm 2.96$ & $252 \%$ & $186^{\mathrm{d}} \pm 1.51$ & $195 \%$ & $160^{b} \pm 2.7$ & $168 \%$ & $196^{\mathrm{c}} \pm 1.14$ & $205 \%$ & $177^{c} \pm 0.7$ & $186 \%$ \\
\hline & 30 days & $98^{\mathrm{a}} \pm 1.51$ & $100 \%$ & $360^{f} \pm 2.07$ & $367 \%$ & $225^{\mathrm{d}} \pm 1.81$ & $229 \%$ & $192^{\mathrm{b}} \pm 1.58$ & $195 \%$ & $231^{\mathrm{c}} \pm 1.87$ & $235 \%$ & $210^{c} \pm 1.92$ & $214 \%$ \\
\hline \multirow{4}{*}{ 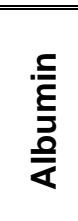 } & Zero Time & $4.1^{a} \pm 0.08$ & $100 \%$ & $4.23^{b} \pm 0.1$ & $103 \%$ & $4.0^{b} \pm 0.19$ & $97 \%$ & $4.1^{a} \pm 0.082$ & $100 \%$ & $4.09^{\mathrm{a}} \pm 0.07$ & $100 \%$ & $4.1^{\mathrm{a}} \pm 0.13$ & $100 \%$ \\
\hline & 10 days & $4.18 a \pm 0.02$ & $100 \%$ & $3.3^{f} \pm 0.07$ & $78 \%$ & $3.9^{d} \pm 0.01$ & $93 \%$ & $4.0^{b} \pm 0.011$ & $95 \%$ & $3.81^{c} \pm 0.02$ & $92 \%$ & $3.95^{c} \pm 0.02$ & $94 \%$ \\
\hline & 20 days & $4.12^{\mathrm{a}} \pm 0.02$ & $100 \%$ & $3.1^{f} \pm 0.02$ & $74 \%$ & $3.62^{d} \pm 0.03$ & $87 \%$ & $3.85^{b} \pm 0.03$ & $93 \%$ & $3.55^{c} \pm 0.02$ & $86 \%$ & $3.7^{c} \pm 0.01$ & $89 \%$ \\
\hline & 30 days & $4.08^{a} \pm 0.01$ & $100 \%$ & $2.7^{f} \pm 0.05$ & $65 \%$ & $3.4^{d} \pm 0.03$ & $84 \%$ & $3.71^{b} \pm 0.018$ & $90 \%$ & $3.3^{c} \pm 0.02$ & $80 \%$ & $3.51^{c} \pm 0.01$ & $86 \%$ \\
\hline
\end{tabular}


Data in Table (2) showed that albumin levels in negative control (100\%) was reduced to $(65 \%)$ in the positive control ( $\mathrm{Pb}$ toxicity), after 30 days of treatment, this decrease in serum albumin may be due to the effect of lead toxicity and cirrhosis of liver cells, which has lost the ability of RNA and protein synthesize.

The treated group one of (300 and 600 $\mathrm{mg} / \mathrm{kg} /$ Watery or acetone extracts) showed an increased in albumin values its comparing to positive control in most of tested groups. The present results are in a good agreement with obtained by Nabil et al (2011) and Bukola et al (2015).

They proved that there was marked decrease in the serum albumin content of rats poisoned by $\mathrm{Pb}$ when compared with that of the normal control rats, whereas feed supplemented with medical plants could significantly correct this metabolic disturbance.

- Effect of moringa leaves extracts on kidneys function test in rats against $\mathrm{Pb}$ Toxicity.

- Blood urea level.

Table (3) show that there is ahighly increased in serum urea and creatinine concentration as aresult of $\mathrm{Pb}$ toxicity on rats (positive controls) may be due to depletion of serum protein. The breakdown of amino acids and deamination that takes place to the formation of large amount of ammonia, and there fore an increase in ammonia circulation which is eventually converted to urea. Addition of water or acetone extracts of moringa leaves to the diet of rats poisoned by $\mathrm{Pb}$ readjusted the level of serum elevated urea and creatinine as compared to positive controls.

\section{Effect of moringa oleifera leaves extract on oxidative stress in rats poisoned by $\mathrm{Pb}$.}

Data in Table (4) showed SOD and CAT activities in all groups, it can be noticed that, the negative control group showed the lowest values $(50.9 \mathrm{U} / \mathrm{mL}$ ) and (4.45) after 30 days, for SOD and CAT respectively while, the positive controls afforded the highest values $(124.54 \mathrm{U} / \mathrm{mL})$ and (9.91 U/L) for SOD and CAT respectively after 30 days of treatment. The addition of one of (300 and 600 $\mathrm{mg} / \mathrm{kg}$ ) watery or acetone extracts to poisoned group by $\mathrm{Pb}$ revealed significant reduction in SOD and CAT activity comparing with Positive controls. The obtained results agreed with those found by Debosree et al., (2012). they demonstrated that the effectiveness of the antioxidant activities of moringa oleifera extracts on protecting against oxidative stress may be attributed to plant phenolics direct reactivity towards reactive oxygen species (ROS) caused by $\mathrm{Pb}$ toxicity .

The data show also that $(600 \mathrm{mg} / \mathrm{kg}$ watery extracts) group exhibited the highest effect for SOD and CAT activities comparing with all treated groups.

\section{Malondialdehyde Level (MDA):}

Data in Table (5) show that the positive control exhibited the highest values, The addition of one of (300 and $600 \mathrm{mg} / \mathrm{kg}$ ) watery or acetone extracts to poisoned groups by $\mathrm{Pb}$ revealed significant reduce in MDA level which recorded $0.361,0.342,0.373$ and 0.340 $\mathrm{U} / \mathrm{mL}$ after 30 days respectively.

\section{Level of lead in organs rats poisoned by $\mathrm{Pb}$.}

Table (6) showed $\mathrm{Pb}$ levels in liver and brain tissues. it can be noticed that, the negative control group showed the lowest values ( 0.31 and $0.15 \mathrm{ug} / \mathrm{g}$ tissue) for liver and brain after 30 days, respectively comparing with Positive control (2.91 and $0.79 \mathrm{ug} / \mathrm{g}$ tissue) for liver and brain respectively 
Table (3): Effect of moringa leaves extracts against urea and creatinine in rats poisoned by $\mathrm{Pb}$.

\begin{tabular}{|c|c|c|c|c|c|c|c|c|c|c|c|c|c|c|c|c|}
\hline \multirow[b]{4}{*}{ Treatments } & \multicolumn{8}{|c|}{ Urea } & \multicolumn{8}{|c|}{ Creatinine } \\
\hline & \multicolumn{8}{|c|}{ Exposure period } & \multicolumn{8}{|c|}{ Exposure period } \\
\hline & \multicolumn{2}{|c|}{ Zero Time } & \multicolumn{2}{|c|}{10 days } & \multicolumn{2}{|c|}{20 days } & \multicolumn{2}{|c|}{30 days } & \multicolumn{2}{|c|}{ Zero Time } & \multicolumn{2}{|c|}{10 days } & \multicolumn{2}{|r|}{20 days } & \multicolumn{2}{|c|}{30 days } \\
\hline & Result & $\%$ & Result & $\%$ & Result & $\%$ & Result & $\%$ & Result & $\%$ & Result & $\%$ & Result & $\%$ & Result & $\%$ \\
\hline $\begin{array}{c}\text { (I) } \\
\text { Negative } \\
\text { control }\end{array}$ & $\begin{array}{c}17^{\mathrm{a}} \\
\pm 0.83\end{array}$ & $100 \%$ & $\begin{array}{c}20^{\mathrm{a}} \\
\pm 0.83\end{array}$ & $100 \%$ & $\begin{array}{c}22^{\mathrm{a}} \\
\pm 1.14\end{array}$ & $100 \%$ & $\begin{array}{r}25^{\mathrm{a}} \\
\pm 1.51\end{array}$ & $100 \%$ & $\begin{array}{c}0.4^{\mathrm{a}} \\
\pm 0.83\end{array}$ & $100 \%$ & $\begin{array}{l}0.49^{\mathrm{a}} \\
\pm 0.04\end{array}$ & $100 \%$ & $\begin{array}{l}0.67^{\mathrm{a}} \\
\pm 0.02\end{array}$ & $100 \%$ & $\begin{array}{l}0.76^{\mathrm{a}} \\
\pm 0.01\end{array}$ & $100 \%$ \\
\hline $\begin{array}{c}\text { ( II ) } \\
\text { Positive control }\end{array}$ & $\begin{array}{r}15^{\mathrm{a}} \\
\pm 1.64\end{array}$ & $\begin{array}{l}88 \\
\%\end{array}$ & $\begin{aligned} & 34^{f} \\
\pm & 0.83\end{aligned}$ & $\begin{array}{l}170 \\
\%\end{array}$ & $\begin{array}{r}44^{f} \\
\pm 1.58\end{array}$ & $\begin{array}{l}200 \\
\%\end{array}$ & $\begin{array}{l}66^{f} \\
\pm 1.3\end{array}$ & $\begin{array}{l}264 \\
\%\end{array}$ & $\begin{array}{l}0.45^{\mathrm{a}} \\
\pm 0.05\end{array}$ & $\begin{array}{l}112 \\
\%\end{array}$ & $\begin{array}{c}1.01^{\dagger} \\
\pm 0.03\end{array}$ & $\begin{array}{l}206 \\
\%\end{array}$ & $\begin{array}{r}1.2^{f} \\
\pm 0.07\end{array}$ & $\begin{array}{l}179 \\
\%\end{array}$ & $\begin{array}{l}1.63^{f} \\
\pm 0.02\end{array}$ & $\begin{array}{c}214 \\
\%\end{array}$ \\
\hline $\begin{array}{c}\text { ( III ) } \\
\text { water } \\
\text { extract300 } \\
\text { mg/kg bw }\end{array}$ & $\begin{array}{l}16^{\mathrm{a}} \\
\pm 1.3\end{array}$ & $\begin{array}{l}94 \\
\%\end{array}$ & $\begin{array}{r}28^{\mathrm{d}} \\
\pm 0.83\end{array}$ & $\begin{array}{c}140 \\
\%\end{array}$ & $\begin{array}{l}31^{d} \\
\pm 1.58\end{array}$ & $\begin{array}{c}140 \\
\%\end{array}$ & $\begin{array}{c}45^{\mathrm{d}} \\
\pm 1.22\end{array}$ & $\begin{array}{c}180 \\
\%\end{array}$ & $\begin{array}{r}0.4^{\mathrm{a}} \\
\pm 0.08\end{array}$ & $\begin{array}{l}100 \\
\%\end{array}$ & $\begin{array}{l}0.87^{d} \\
\pm 0.01\end{array}$ & $\begin{array}{c}177 \\
\%\end{array}$ & $\begin{array}{l}1.01^{d} \\
\pm 0.03\end{array}$ & $\begin{array}{c}150 \\
\%\end{array}$ & $\begin{array}{l}1.21^{\mathrm{d}} \\
\pm 0.01\end{array}$ & $\begin{array}{c}159 \\
\%\end{array}$ \\
\hline $\begin{array}{c}\text { ( IV ) } \\
\text { water extract } \\
600 \mathrm{mg} / \mathrm{kg} \text { bw }\end{array}$ & $\begin{array}{c}18^{\mathrm{a}} \\
\pm 0.83\end{array}$ & $\begin{array}{l}105 \\
\%\end{array}$ & $\begin{array}{r}24^{\mathrm{b}} \\
\pm 1.00\end{array}$ & $\begin{array}{l}120 \\
\%\end{array}$ & $\begin{array}{r}26^{\mathrm{b}} \\
\pm 1.14\end{array}$ & $\begin{array}{l}118 \\
\%\end{array}$ & $\begin{array}{c}35^{\mathrm{b}} \\
\pm 1.14\end{array}$ & $\begin{array}{c}140 \\
\%\end{array}$ & $\begin{array}{l}0.48^{\mathrm{b}} \\
\pm 0.05\end{array}$ & $\begin{array}{l}120 \\
\%\end{array}$ & $\begin{array}{l}0.74^{b} \\
\pm 0.01\end{array}$ & $\begin{array}{l}151 \\
\%\end{array}$ & $\begin{array}{c}0.81^{b} \\
\pm 0.02\end{array}$ & $\begin{array}{l}120 \\
\%\end{array}$ & $\begin{array}{l}0.96^{\mathrm{b}} \\
\pm 0.01\end{array}$ & $\begin{array}{c}126 \\
\%\end{array}$ \\
\hline $\begin{array}{c}\text { ( V ) } \\
\text { acetone extract } \\
300 \mathrm{mg} / \mathrm{kg} \mathrm{bw}\end{array}$ & $\begin{array}{l}18^{\mathrm{a}} \\
\pm 0.7\end{array}$ & $\begin{array}{c}105 \\
\%\end{array}$ & $\begin{array}{c}30^{\mathrm{e}} \\
\pm 0.83\end{array}$ & $\begin{array}{c}150 \\
\%\end{array}$ & $\begin{array}{c}35^{\mathrm{e}} \\
\pm 1.58\end{array}$ & $\begin{array}{c}159 \\
\%\end{array}$ & $\begin{array}{c}50^{\mathrm{e}} \\
\pm 1.30\end{array}$ & $\begin{array}{c}200 \\
\%\end{array}$ & $\begin{array}{l}0.54^{b} \\
\pm 0.03\end{array}$ & $\begin{array}{c}135 \\
\%\end{array}$ & $\begin{array}{l}0.95^{\mathrm{e}} \\
\pm 0.01\end{array}$ & $\begin{array}{c}193 \\
\%\end{array}$ & $\begin{array}{l}1.09^{\mathrm{e}} \\
\pm 0.02\end{array}$ & $\begin{array}{c}162 \\
\%\end{array}$ & $\begin{array}{l}1.28^{\mathrm{e}} \\
\pm 0.02\end{array}$ & $\begin{array}{c}168 \\
\%\end{array}$ \\
\hline $\begin{array}{c}\text { (VI) } \\
\text { acetone extract } \\
600 \mathrm{mg} / \mathrm{kg} \mathrm{bw}\end{array}$ & $\begin{array}{r}17^{\mathrm{a}} \\
\pm 1.14\end{array}$ & $\begin{array}{l}100 \\
\%\end{array}$ & $\begin{array}{c}27^{c} \\
\pm 0.83\end{array}$ & $\begin{array}{c}135 \\
\%\end{array}$ & $\begin{array}{r}28^{c} \\
\pm 1.17\end{array}$ & $\begin{array}{l}127 \\
\%\end{array}$ & $\begin{array}{r}46^{c} \\
\pm 1.3\end{array}$ & $\begin{array}{l}184 \\
\%\end{array}$ & $\begin{array}{r}0.4^{\mathrm{a}} \\
\pm 0.03\end{array}$ & $\begin{array}{l}100 \\
\%\end{array}$ & $\begin{array}{l}0.81^{\mathrm{c}} \\
\pm 0.01\end{array}$ & $\begin{array}{c}165 \\
\%\end{array}$ & $\begin{array}{r}0.91^{\mathrm{c}} \\
\pm 0.01\end{array}$ & $\begin{array}{l}135 \\
\%\end{array}$ & $\begin{array}{l}1.08^{\mathrm{c}} \\
\pm 0.01\end{array}$ & $\begin{array}{c}142 \\
\%\end{array}$ \\
\hline
\end{tabular}

The same letters in each column represents the insignificant different at $(p \geq 0.5)$ 
Table (4): Effect of moringa leaves extracts against SOD and CAT activity in rats poisoned by Pb.

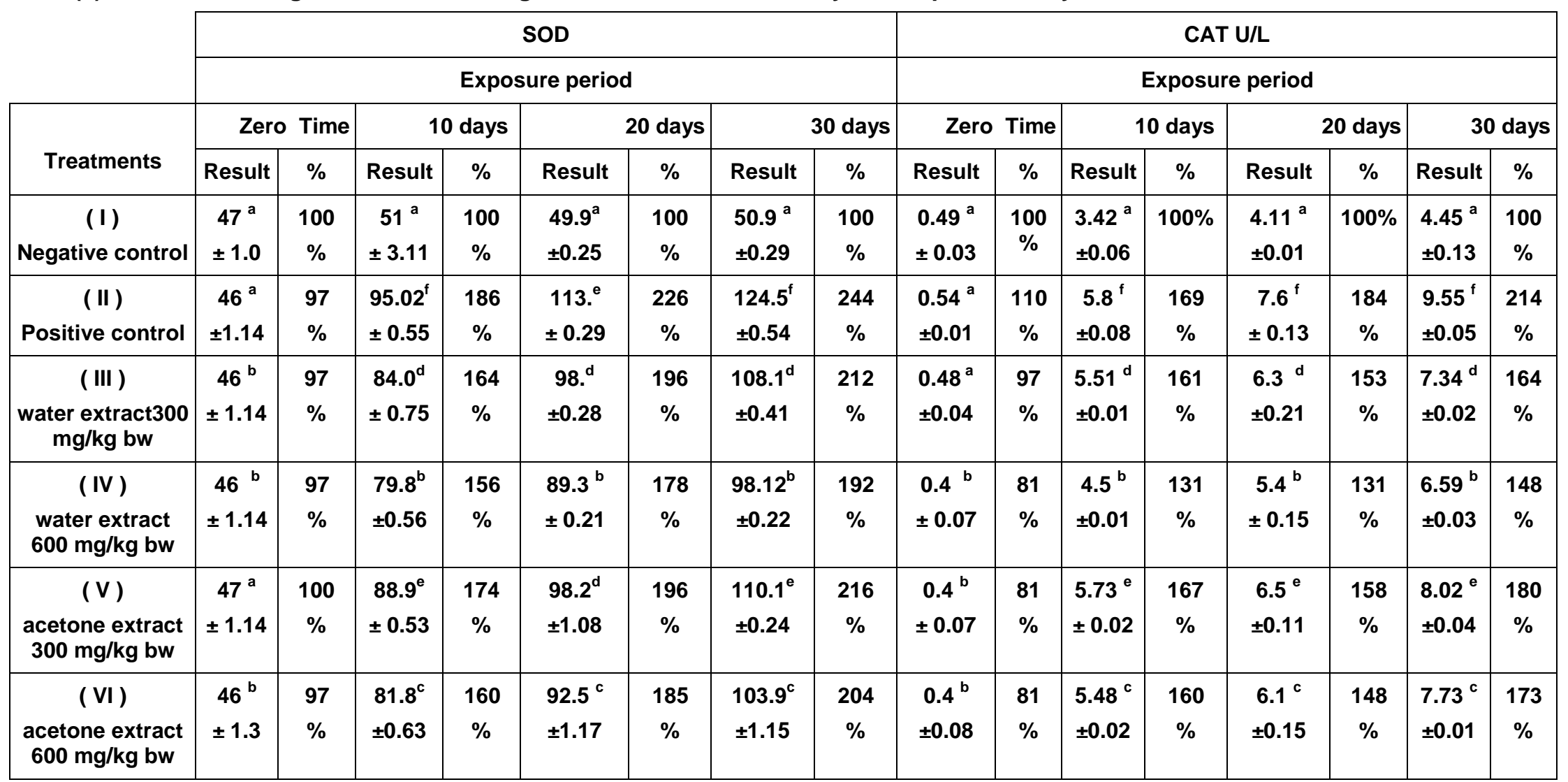

The same letters in each column represents the insignificant different at $(p \geq 0.5)$ 
Table (5): Effect of moringa leaves extracts against MDA activity in rats poisoned by $\mathrm{Pb}$.

\begin{tabular}{|c|c|c|c|c|c|c|c|c|}
\hline \multirow[b]{4}{*}{ Treatments } & \multicolumn{8}{|c|}{ MDA nmol/ml } \\
\hline & \multicolumn{8}{|c|}{ Exposure period } \\
\hline & \multicolumn{2}{|c|}{ Zero Time } & \multicolumn{2}{|c|}{10 days } & \multicolumn{2}{|l|}{20 days } & \multicolumn{2}{|c|}{30 days } \\
\hline & Result & $\%$ & Result & $\%$ & Result & $\%$ & Result & $\%$ \\
\hline ( I ) Negative control & $0.106^{a} \pm 0.001$ & $100 \%$ & $0.112^{a} \pm 0.003$ & $100 \%$ & $0.101^{\mathrm{a}} \pm 0.004$ & $100 \%$ & $0.110^{a} \pm 0.001$ & $100 \%$ \\
\hline ( II ) Positive control & $0.099^{b} \pm 0.007$ & $93 \%$ & $0.314^{f} \pm 0.017$ & $280 \%$ & $0.389^{\mathrm{e}} \pm 0.001$ & $385 \%$ & $0.420^{f} \pm 0.001$ & $381 \%$ \\
\hline ( III ) water extract300 mg/kg bw & $0.098^{b} \pm 0.005$ & $93 \%$ & $0.283^{d} \pm 0.002$ & $252 \%$ & $0.311^{c} \pm 0.001$ & $307 \%$ & $0.361^{d} \pm 0.001$ & $328 \%$ \\
\hline ( IV )water extract $600 \mathrm{mg} / \mathrm{kg} \mathrm{bw}$ & $0.106^{a} \pm 0.005$ & $100 \%$ & $0.252^{b} \pm 0.003$ & $225 \%$ & $0.291^{b} \pm 0.001$ & $288 \%$ & $0.342^{b} \pm 0.001$ & $310 \%$ \\
\hline ( V )acetone extract $300 \mathrm{mg} / \mathrm{kg}$ bw & $0.107^{\mathrm{a}} \pm 0.001$ & $101 \%$ & $0.292^{e} \pm 0.001$ & $260 \%$ & $0.322^{d} \pm 0.01$ & $318 \%$ & $0.373^{e} \pm 0.001$ & $340 \%$ \\
\hline ( VI ) acetone extract $600 \mathrm{mg} / \mathrm{kg} \mathrm{bw}$ & $0.098^{b} \pm 0.005$ & $93 \%$ & $0.274^{c} \pm 0.001$ & $244 \%$ & $0.311^{c} \pm 0.001$ & $307 \%$ & $0.340^{c} \pm 0.006$ & $309 \%$ \\
\hline
\end{tabular}

Table (6): Effect of moringa leaves extracts on organs rats poisoned by $\mathrm{Pb}$.

\begin{tabular}{|c|c|c|c|}
\hline & & \multicolumn{2}{|c|}{ Concentration of lead in liver and brain tissue } \\
\hline \multicolumn{2}{|r|}{ Treatments } & Liver Tissue & brain Tissue \\
\hline$(1)$ & Negative control & $0.31^{a} \pm 0.012$ & $0.15^{a} \pm 0.012$ \\
\hline ( II ) & Positive control & $2.91^{f} \pm 0.020$ & $0.79^{f} \pm 0.016$ \\
\hline ( III ) & water extract300 mg/kg bw & $2.11^{d} \pm 0.028$ & $0.61^{d} \pm 0.019$ \\
\hline ( IV ) & water extract $600 \mathrm{mg} / \mathrm{kg} \mathrm{bw}$ & $1.88^{b} \pm 0.048$ & $0.21^{b} \pm 0.016$ \\
\hline ( v ) & acetone extract $300 \mathrm{mg} / \mathrm{kg}$ bw & $2.22^{e} \pm 0.062$ & $0.72^{e} \pm 0.012$ \\
\hline ( VI ) & acetone extract $600 \mathrm{mg} / \mathrm{kg} \mathrm{bw}$ & $2.11^{c} \pm 0.009$ & $0.22^{c} \pm 0.023$ \\
\hline
\end{tabular}

The same letters in each column represents the insignificant different at $(p \geq 0.5)$ 
The treatment with Moringa leaf extracts resulted in a decrease in the accumulation of lead in both liver and brain in rats.

\section{REFERENCES}

A.O.A.C. Association of Official Analytical Chemists (2000). Official Methods of Analysis, 17th ed. of the Association of Official Analytical Chemistry, Washington, D.C, MSA.

Adhikari, N., N. Sinha and R. Narayan (2001). Lead-Induced Cell Death in Testes of Young Rats. Journal of Applied Toxicology, 21: 275-277. http://dx.doi.org/10.1002/jat.754.

Aebi, H. (1984). Methods in Enzymatic Analysis. Academic Press. New York. 3: 276-286.

Borges, E. M., M. R. Carvalho, V. A. Neves, M. A. Silva and L. ArantesPereira (2014). Chemical characteristics and fractionation of proteins from Moringa oleifera Lam. leaves. Food. Chem., 14: 51-54.

Bukola, R. O., A. A. Adeleke, M. I. Omamuyovwi and $O$. A. Stephen (2015). Lead-induced damage on hepatocytes and hepatic reticular fibres in rats; protective role of aqueous extract of moringa oleifera leaves (lam) Journal of Biosciences and Medicines, 3: 27-35.

Cannon, D.C., I. Olitzky and J.A. Inkpen (1974). Proteins. In: Clinical chemistry, principles and technics. 2-nd ed. RJ Henery. DC Cannon, JW Winkelman, editors, Harper and Row, New York, pp 407- 421.

Clayton, C.A., E.D. Pellizzari and J.J. Quackenboss (2002). National Human Exposure Assessment Survey: Analysis of Exposure Pathways and Routes for Arsenic and Lead in EPA Region 5. Journal of Exposure Analysis and Environmental Epidemiology, 12: 29-43. http:/l dx.doi.org /10.1038/sj.jea .7500198)
Dahot, M. U. (1988). Vitamin contents of flowers and seeds of Moringa oleifera. Pakistan Journal of Biochemistry and Molecular Biology, 21: 1-24.

Daniel, H.D. and C.M. George (1972). Peach seed dormancy in relation to indogenous inhibitors and applied growth substances. J. Amer. Soc. Hort. Sci., 97(5): 651-654.

Dewanto, V., X. Wu, K. K. Adom and R.H. Liu (2002). Thermal processing enhances the nutritional value of tomatoes by increasing total antioxidant activity. J Agric Food Chem. 50: 3010-3014.

Debosree, G., B. F. Syed, M. Elina, D. Monalisa and Debasish (2012). protective effect of aqueous leaf extract of murraya koenigi against lead induced oxidative stress in rat liver, heart and kidney: a dose response study. Asian J Pharm Clin Res, 5 (Suppl 4): 54-58.

Gulcin, I., M. Oktay, I. Kufrevioglu and A. Aslan (2002). Determination of antioxidant activity of lichen Cetraria islandica (L.) Ach. J. Ethnopharmacol. 79: 325-29.

Hegested, M., R. S. Mills, G. A. Elvhjem and E.B. Hart (1941). Choline in the nutrition of chicks. J. Biochem, 138:459.

Hossain, M. D., A. Sadika, M. D. L. Aminul, A. B. Mohiuddin and $S$. Mohammad (2012). In vitro antioxidant activity of moringa oleifera leaves, s. J. Pharm. Sci. 5(I\&2): 4I-52.

Landue, S. and B.S. Everitt (2004). A Handbook of statistical Analyses using SPSS. -London: Chapman \&Hall/CRC press LLC, $337 \mathrm{p}$.

Moss, D.W., A.R. Henderson and J.F. Kachmar (1987). Enzymes in: Tietz NW, ed. Fundamentals of clinical chemistry. 3 rd ed. Philadelphia: WB Saunders; 346-421.

Murrey, R.K., D.K. Granner, V.W. Rodwell (2006). Harper's illustrate biochemistry. 27th ed. Boston, New 
York, Singapore: McGrow Hil Comp. Inc.

Nabil, M. I., A. E. Esam, H. S. El-Belt and Yasmin E. A. (2011). The Effect of Lead Acetate Toxicity on Experimental Male Albino Rat, Biol Trace Elem Res (2011) 144:1120-1132, DOI 10.1007/s12011-011-9149-z.

Nishikimi, M., N.A. Roa and K. Yogi (1972). Measurement of superoxide dismutase. Biochem. Biophys. Res. Common, 46:849-854

Ohkawa, H., W. Ohishi and K. Yagi (1979). Assay for lipid peroxides in animal tissues by thiobarbituric acid reaction, Anal. Biochem. 95: 351-358.

Oyaizu, M. (1986). Studies on product of browning reaction prepared from glucose amine. Jpn J Nutr. 07:307-15.

Pirjo, P.S. and E.K. Pekka (1996). Determination of protein in foods: comparison of net protein and crude protein $(\mathrm{N} \times 6.25)$ values. Food chemistry. 57(1): 27-31.

Paliwal, R., V. Sharma, A. Roloff, H. Weisgerber, U. Lang, B. Stimm, V. G. Wiley-Vch and P. Janmeda (2011). A review on horse radish tree (Moringa oleifera): A multipurpose tree with high economic and commercial importance. Asian J. Biotechnol. 3: 317-328.

Schneeman, B. O., R. Rice and B.D. Richter (1989). Reduction of plasma and hepatic triacylglycerids with whole milk containing diets in rats. J. Nutr., 119:965-970.

Shalan, M. G., M. S. Mostafa, M. M. Hassouna, S. E. Hassab El-Nabi and A. El-Rafaie (2005). Amelioration of lead toxicity on rat liver with vitamin C and silymarin supplements. Toxicology, 206: 1-15.

White, L.D., D.A. Cory-Slechta, M.E. Gilbert, E. Tiffany-Castiglioni, N.H. Zawia, M. Virgolini, Rossi-George and S.M. Lasley (2007)."New and evolving concepts in the neurotoxicology of lead". Toxicology and applied pharmacology 225 (1): 1-27.)

Young, D.S. (1990). Effects of drugs on clinical laboratory tests. Third edition 4th Ed. Washington, DC; AACC Press: 3:6 -12.

Young, D.S. (2001). Effects of disease on clinical lab. Tests. 4th ed AACC American Association for Clinical Chemistry. Zargary, A (1989): The medicinal plants.5th. Ed. Tehran university publication, Tehran. 312314.

Zuo, Y., H. Chen and Y. Deng (2002). Simultaneous Determination of Catechins Caffeine and Gallic acids in Green, Oolong, Black and Pu-err Teas using HPLC with a Photodiode Array Detector. Talanta, 57: 307-316.

\section{تاثير مستخلصات اوراق المورينجا كمضاد للتشمم بالرصاص فى القئران}


كمال إمام حسين ، سامية محمود خليل ، فيصل عبدالحفيظ عبدالسلام

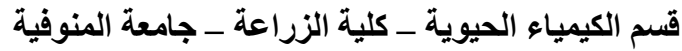

الملخص العربى المذي

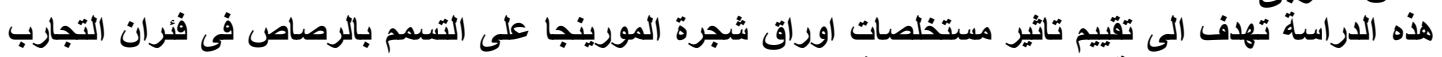

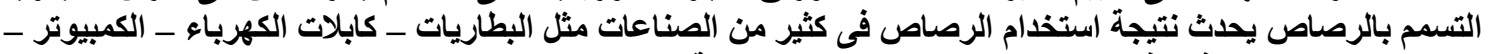

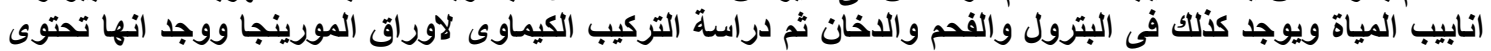

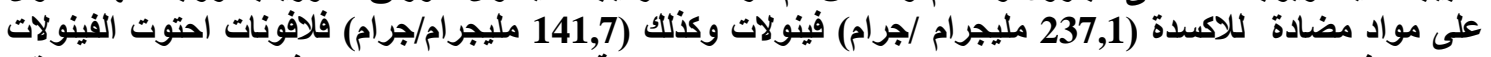

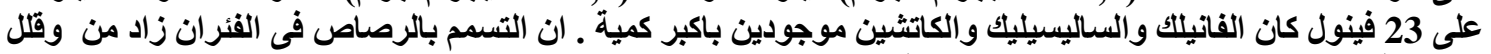

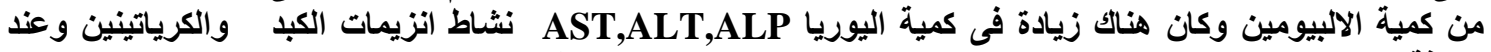

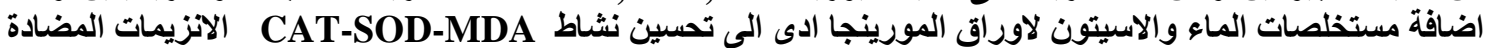

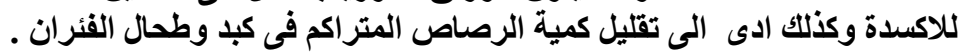

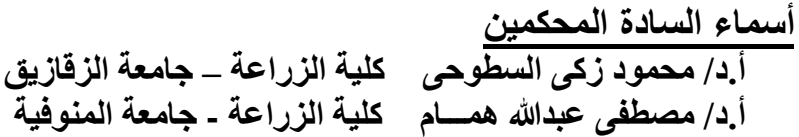

